\title{
NEIGHBOURHOOD SOCIAL CHARACTERISTICS AND RISK OF VERY PRETERM BIRTH IN A FRENCH AND ENGLISH REGION
}

\author{
M. Bonet ${ }^{1}$, L.K. Smith ${ }^{2}$, E.S. Draper ${ }^{2}$, J. Zeitlin ${ }^{1}$ \\ ${ }^{I}$ Epidemiological Research Unit on Perinatal and Women's and Children's Health, INSERM U953, Paris, \\ France, ${ }^{2}$ Department of Health Science, University of Leicester, Leicester, UK
}

Background and aim: Social factors affect the risk of very preterm birth, but the underlying mechanisms are not well understood. We assessed the impact of neighborhood characteristics on the risk of very preterm birth by pregnancy complication.

Methods: Infants from the MOSAIC population-based cohort of births between 22 and 31 weeks of gestation in 2003 from two English and French regions $(\mathrm{N}=1744)$ were geocoded to their neighbourhood census tracts. Neighbourhoods were grouped into quartiles by socioeconomic characteristics (unemployment rate, proportion manual workers, proportion with high school education only) based on the place of residence of infants enumerated in the census. We derived odds ratios for the risk of preterm birth by multiplicity, gestational age at delivery ( $<=27$ 28-29 30-31 weeks) and medical complication (hypertension, fetal growth restriction, antepartum haemorrhage, preterm rupture of membranes, preterm labour).

Results: Neighbourhood social characteristics were related to increased risk of preterm birth for singletons in both countries with odds ratios ranging between 2.1 and 2.4 in the most deprived versus least deprived quartiles. Twins were not more likely to live in poor neighbourhoods than census infants. Among very preterm singleton infants, there was no evidence of a relationship between neighbourhood characteristics and gestational age or medical complication.

Conclusions: In both regions, neighbourhood social factors had a significant impact on the risk of singleton very preterm birth, but not twin very preterm births. Estimates of the risk of singleton preterm birth associated with living in poorer neighbourhoods were similar by pregnancy complications. 